\title{
Approximate Capacity Subregions of Uniform Multihop Wireless Networks
}

\author{
Peng-Jun Wan, and Lixin Wang \\ Department of Computer Science \\ Illinois Institute of Technology \\ Emails: wan@cs.iit.edu,wanglix@iit.edu
}

\author{
Ai Huang, Minming Li, and Frances Yao \\ Department of Computer Science \\ City University of Hong Kong \\ Emails: \{ahuang, mli000, csfyao\}@ cityu.edu.hk
}

\begin{abstract}
The capacity region of multihop wireless network is involved in many capacity optimization problems. However, the membership of the capacity region is NP-complete in general, and hence the direct application of capacity region is quite limited. As a compromise, we often substitute the capacity region with a polynomial approximate capacity subregion. In this paper, we construct polynomial $\mu$-approximate capacity subregions of multihop wireless network under either 802.11 interference model or protocol interference model in which all nodes have uniform communication radii normalized to one and uniform interference radii $\rho \geq 1$. The approximation factor $\mu$ decreases with $\rho$ in general and is smaller than the best-known ones in the literature. For example, $\mu=3$ when $\rho \geq 2.2907$ under the 802.11 interference model or when $\rho \geq 4.2462$ under the protocol interference model. Our construction exploits a nature of the wireless interference called strip-wise transitivity of independence discovered in this paper and utilize the independence polytopes of cocomparability graphs in a spatial-divide-conquer manner. We also apply these polynomial $\mu$-approximate capacity subregions to compute $\mu$-approximate solutions for maximum (concurrent) multiflows.
\end{abstract}

Index Terms-Capacity region, maximum (concurrent) multiflows, approximation algorithm

\section{INTRODUCTION}

A multihop wireless network $\mathbf{N}$ is specified, in its most general format, by a triple $(V, A, \mathcal{I})$, where $V$ is the set of networking nodes, $A$ is the set of communication links among $V$, and $\mathcal{I}$ is the collection of sets of independent (or conflictfree) links in $A$ specified implicitly by an interference model. The communication topology of $\mathbf{N}$ is the digraph $(V, A)$. The capacity region of $\mathbf{N}$ is the convex hull $P \subset \mathbb{R}_{+}^{A}$ of the incidence vectors of all sets in $\mathcal{I}$. Alternatively, the capacity region of $\mathbf{N}$ can also be defined in terms of link schedules. A (fractional) link schedule $\Pi$ in $\mathbf{N}$ is a set

$$
\left\{\left(I_{j}, \lambda_{j}\right) \in \mathcal{I} \times \mathbb{R}_{+}: 1 \leq j \leq k\right\} ;
$$

the two values $k$ and $\sum_{j=1}^{k} \lambda_{j}$ are referred to as the size and length (or latency) of $\Pi$ respectively. Any link schedule $\Pi$ in $\mathbf{N}$ of length at most one determines a link capacity function $c_{\Pi} \in \mathbb{R}_{+}^{A}$ given by

$$
c_{\Pi}(e)=\sum_{1 \leq j \leq k} \lambda_{j}\left|I_{j} \cap\{e\}\right|
$$

for each $e \in A$; and $\Pi$ is said to be a link schedule for $c_{\Pi}$. Then, the capacity region $P$ of $\mathbf{N}$ consists of link capacity functions determined by link schedules in $\mathbf{N}$ of length at most one.

The capacity region is involved in many capacity optimization problems. However, the membership of the capacity region is NP-complete in general [7], and hence the direct application of capacity region is quite limited. As a compromise, we often substitute the capacity region $P$ with a subset $Q$ of $P$ which satisfies the three conditions: (1) $Q$ has an explicit representation by a polynomial number of linear inequalities, (2) there is a polynomial algorithm which produces a link schedule of length at most one for any $d \in Q$, and (3) $P \subseteq \mu Q$ for some $\mu \geq 1$. The first condition ensures the membership of $Q$ is polynomial, the second condition implies that $Q \subseteq P$, and the third condition ensures that $Q$ is "close" to $P$. A subset $Q$ of $P$ satisfying these three conditions is referred to as a polynomial $\mu$-approximate capacity subregion.

The polynomial constant-approximate capacity subregion plays a central role in the design and analysis of polynomialtime constant-approximation algorithms for capacity optimization problems such as Maximum Multiflow (MMF) and Maximum Concurrent Multiflow (MCMF). Suppose that we are given a set of commodities in a network N. For any link schedule $\Pi$ in $\mathbf{N}$ of length at most one, the maximum multiflow of these commodities subject to the capacity function $c_{\Pi}$ is referred to as the maximum multiflow subject to $\Pi$. Suppose in addition that each commodity also has a demand associated with it. For any link schedule $\Pi$ in $\mathbf{N}$ of length at most one, the maximum concurrent multiflow of these commodities subject to the capacity function $c_{\Pi}$ is referred to as the maximum concurrent multiflow subject to $\Pi$. Given a set of commodities in a specified network $\mathbf{N}$, the problem MMF seeks a link schedule $\Pi$ in $\mathbf{N}$ of length at most one such that the maximum multiflow subject to $\Pi$ is maximized. Given a set of commodities with demands in a specified network $\mathbf{N}$, the problem MCMF seeks a link schedule $\Pi$ in $\mathbf{N}$ of length at most one such that the maximum concurrent multiflow 
subject to $\Pi$ is maximized. It was shown in [7] that if we can find a polynomial $\mu$-approximate capacity subregion, then both MMF and MCMF admit a polynomial $\mu$-approximation.

In this paper we construct polynomial constant-approxmatie capacity subregions of multihop wireless networks under either the 802.11 interference model or the protocol interference model respectively with uniform communication radii normalized to one and uniform interference radii equal to $\rho \geq 1$. The communication (respectively, interference) range of a node $v \in V$ is the disk centered at $v$ of radius one and $\rho$ respectively. These networks are collectively referred to as uniform multihop wireless networks. Under both interference models, an instance of a network $\mathbf{N}$ is specified by a finite planar set $V$ of nodes and the value of the interference radius $\rho$. The set $A$ of communication links consists of all pairs $(u, v)$ satisfying that the Euclidean distance between $u$ and $v$, denoted by $\|u v\|$, is at most one. Under the 802.11 interference model, two links in $A$ conflict with each other if and only if at least one link has an end lying in the interference range of some endpoint of the other link; under the protocol interference model, two links in $A$ conflict with each other if and only if the receiving end of at least one link lies in the interference range of the transmitting end of the other link. Under either of the two interference models, a set $I$ of links in $A$ are independent (i.e., $I \in \mathcal{I}$ ) if all links in $I$ are mutually conflict-free. The conflict graph of $A$ is the undirected graph on $A$ in which two links are adjacent if and only if they conflict with each other. So, $\mathcal{I}$ is essentially the collection of the independent sets in the conflict graph of $A$.

The polynomial constant-approximate capacity subregions of uniform multihop wireless networks under the 802.11 interference model or the protocol interference model have been studied implicitly in [1] [3] [7] [9]. Under the 802.11 interference model, Alicherry et al. [1] gave implicitly a polynomial $\mu$-approximate capacity subregion, where $\mu$ was claimed to grow with $\rho$ in general and equal to 4,8 and 12 respectively when $\rho=1,2,2.5$ respectively. Buragohain et al. [3] discovered that the claim in [1] that $\mu=4$ when $\rho=1$ is wrong and pointed out that $\mu$ should be 8 when $\rho=1$. They further proposed implicitly a polynomial 3approximate capacity subregion regardless of the value of $\rho$. However, their capacity subregion was shown in [7] to contain some element which does not belong to the capacity region. Hence, their capacity subregion is wrong. Wan [7] then constructed implicitly yet another polynomial 7-approximate capacity subregion regardless of $\rho$. Under the protocol interference model with $\rho>1$, Wang et al. [9] proposed implicitly a polynomial $2\left[2 \pi / \arcsin \frac{\rho-1}{2 \rho}\right]$-approximate capacity subregion, which also turned to be false as shown in [7]. Wan [7] then devised implicitly a $2\left(\left\lceil\pi / \arcsin \frac{\rho-1}{2 \rho}\right\rceil-1\right)$ approximate capacity subregion.
The approach used in this paper is totally different from those followed in [1] [3] [7] [9]. Our approach exploits a nature of the wireless interference called strip-wise transitivity of independence under either the 802.11 interference model or the protocol interference model discovered in this paper. Such nature enables us to utilize the independence polytopes of cocomparability graphs [5] in a spatial-divide-conquer manner to build a polynomial $\mu$-approximate capacity subregion. The value of $\mu$ decreases with $\rho$ in general and is smaller than the best-known ones obtained in [7]. For example, $\mu=3$ when $\rho \geq 2.2907$ under the 802.11 interference model or when $\rho \geq 4.2462$ under the protocol interference model. As a result, our polynomial capacity subregions give rise to improved practical approximation algorithms for both MMF and MCMF.

At the end of this section, we introduce some basic notations and terms used throughout this paper. For any $r>0$, a $r$-disk is a disk of radius $r$. In particular, a unit-disk is a disk of radius one. The $r$-disk centered at a node $u$ is denoted by $B_{r}(u)$. For any set $U$ of nodes, the union of the $r$-disks centered at the nodes in $U$ is denoted by $B_{r}(U)$. The vertical line through a point $v$ is denoted by $l_{v}$. Let $S$ be a finite subset. For any real function $f \in \mathbb{R}^{S}$ and any subset $S^{\prime} \subseteq S, f\left(S^{\prime}\right)$ denotes $\sum_{e \in S^{\prime}} f(e)$.

\section{PReliminaries}

Consider a digraph $D=(V, A)$. For each vertex $v \in V$, we use $\delta_{D}^{i n}(v)$ (respectively, $\delta_{D}^{o u t}(v)$ ) to denote the set of links in $D$ entering (respectively, leaving) $v$. Consider two distinct vertices $s, t \in V$. A vector $f \in \mathbb{R}_{+}^{A}$ is called a flow from $s$ to $t$, or simply a $s-t$ flow, if for each $v \in V \backslash\{s, t\}$,

$$
f\left(\delta_{D}^{\text {out }}(v)\right)=f\left(\delta_{D}^{\text {in }}(v)\right)
$$

This condition is called the flow conservation law: the amount of flow entering a vertex $v \neq s, t$ should be equal to the amount of flow leaving $v$. The value of a flow $f$ from $s$ to $t$ is, by definition:

$$
\operatorname{val}(f)=f\left(\delta_{D}^{\text {out }}(s)\right)-f\left(\delta_{D}^{\text {in }}(s)\right) .
$$

So the value is the net amount of flow leaving $s$, which is also equal to the net amount of flow entering $t$.

Let $G=(V, E)$ be an undirected graph. A subset $I$ of $V$ is an independent set of $G$ if no two vertices in $I$ are adjacent. For any $d \in \mathbb{R}_{+}^{V}$, a fractional (weighted) coloring of $(G, d)$ is a set of $k$ pairs $\left(I_{j}, \lambda_{j}\right) \in \mathcal{I} \times \mathbb{R}_{+}$for $1 \leq j \leq k$ satisfying that for each $v \in V$,

$$
\sum_{1 \leq j \leq k, v \in I_{j}} \lambda_{j}=d(v) .
$$


The two values $k$ and $\sum_{j=1}^{k} \lambda_{j}$ are referred to as the number and weight of the fractional coloring respectively. The fractional chromatic number $\chi_{f}(G, d)$ of $(G, d)$ is defined as the minimum weight of all fractional colorings of $(G, d)$. The independence polytope $P$ of $G$ is the convex hull of the incidence vectors of the independent sets in $G$. Equivalently, it consists of all $d \in \mathbb{R}_{+}^{V}$ with $\chi_{f}(G, d) \leq 1$. A polytope $Q$ is said to be a $\mu$-approximation of $P$ for some $\mu \geq 1$ if $Q \subseteq P \subseteq \mu Q$.

A graph $G=(V, E)$ is said to be a cocomparability graph [5] if there is a vertex ordering $\left\langle v_{1}, v_{2}, \cdots, v_{n}\right\rangle$ of $V$ satisfying that if $i<j<k$ and $v_{i} v_{k} \in E$ then either $v_{i} v_{j} \in E$ or $v_{j} v_{k} \in E$. Such ordering is referred to as its cocomparability ordering. Then, a minimum-weighted fractional coloring of $(G, d)$ can be computed in polynomial time [4], [6]; moreover, the independence polytope of $G$ can be represented by a linear number of inequalities [2]. Suppose that $G=(V, E)$ is a cocomparability graph with a cocomparability ordering $\left\langle v_{1}, v_{2}, \cdots, v_{n}\right\rangle$ of its vertices. Let $s$ and $t$ be two vertices not in $V$, and define an auxiliary digraph $G^{*}=\left(V \cup\{s, t\}, A^{*}\right)$ in which

$$
\begin{aligned}
A^{*} & =\left\{\left(v_{i}, v_{j}\right): 1 \leq i<j \leq n, v_{i} v_{j} \notin E\right\} \\
& \cup\{(s, v): v \in V\} \cup\{(v, t): v \in V\} .
\end{aligned}
$$

Its construction can be interpreted as follows: We take the complement graph $G^{c}$ of $G$ and orient each edge $v_{i} v_{j}$ with $i<j$ in $G^{c}$ to an arc $\left(v_{i}, v_{j}\right)$. After that we add an auxiliary source $s$ and an auxiliary sink $t$, and then add an arc from $s$ to every node in $V$ and an arc from every node in $V$ to $t$. Thus, $G^{*}$ is referred to as the augmented complementary digraph of $G$. Clearly, any independent set in $G$ defines a $s-t$ path in $G^{*}$ and vice versa. We use $\mathcal{F}$ to denote the set of $s-t$ flows $f$ in $G^{*}$. For any flow $f \in \mathcal{F}$, we define a function $d_{f} \in \mathbb{R}^{V}$ by $d_{f}(v)=f\left(\delta_{G^{*}}^{o u t}(v)\right)$. Then, the independence polytope of $G$ is

$$
P=\left\{d_{f}: f \in \mathcal{F}, \operatorname{val}(f) \leq 1\right\} .
$$

For any $f \in \mathcal{F}$ with $\operatorname{val}(f) \leq 1$, a link schedule for $d_{f}$ can be computed as follows. We first decompose the flow $f$ into path flows using the standard flow decomposition method [4]. Suppose that

$$
\left\{\left(p_{j}, \lambda_{j}\right): 1 \leq j \leq k\right\}
$$

is a path flow decomposition of $f$. Let $I_{j}$ be the set of vertices other than $s$ and $t$ in the path $p_{j}$ for each $1 \leq j \leq k$. Then, $I_{j}$ is an independent set of $G$. Hence,

$$
\left\{\left(I_{j}, \lambda_{j}\right): 1 \leq j \leq k\right\}
$$

is a link schedule of $d_{f}$.

\section{STRIP-WISE TRANSITIVITY OF INDEPENDENCE}

In this section, we prove a strip-wise transitivity of independence under the 802.11 interference model described in Theorem 1, and a similar strip-wise transitivity of independence under the protocol interference model described in Theorem 2 .

Theorem 1: Consider a multihop wireless network under the 802.11 interference model in which each node has communication radius equal to one and interference radius equal to $\rho$. Suppose that $a_{1}, a_{3}$ and $a_{2}$ are three links whose midpoints lie in a horizontal strip of height

$$
h(\rho)=\sqrt{\rho^{2}-\frac{1}{4}} \cos \left(\frac{\pi}{6}+\arcsin \frac{1}{2 \rho}\right)
$$

from left to right. If both $a_{1}$ and $a_{2}$ are independent with $a_{3}$, then $a_{1}$ and $a_{2}$ are also independent with each other.

Theorem 2: Consider a multihop wireless network under the protocol interference model in which each node has communication radius equal to one and interference radius equal to $\rho$. Suppose that $a_{1}, a_{3}$ and $a_{2}$ are three links whose transmitting endpoints lie in a horizontal strip of height

$$
h(\rho)=(\rho-1) \sin \left(\arccos \frac{\rho-1}{2 \rho}-\arcsin \frac{1}{\rho}\right)
$$

from left to right. If both $a_{1}$ and $a_{2}$ are independent with $a_{3}$, then $a_{1}$ and $a_{2}$ are also independent with each other.

The next two subsections are devoted to the proofs of the above two theorems respectively.

\section{A. Proof of Theorem 1}

The height function $h(\rho)$ defined in Theorem 1 has the following geometric interpretation. Consider four points $v_{1}, u_{1}, u_{2}, v_{2}$ counterclockwise on a circle of radius $\rho$ centered at a point $p$ satisfying that $\left\|u_{1} v_{1}\right\|=\left\|u_{2} v_{2}\right\|=1$ and $\left\|u_{1} u_{2}\right\|=\rho$ (see Fig. 1). Let $z_{1}$ and $z_{2}$ be the midpoints of $u_{1} v_{1}$ and $u_{2} v_{2}$ respectively, and $q$ be the perpendicular foot of $p$ on $z_{1} z_{2}$. Then, $\|p q\|$ is exactly $h(\rho)$. Indeed, in the right triangle $p u_{1} z_{1}$, we have

$$
\left\|p z_{1}\right\|=\sqrt{\rho^{2}-\frac{1}{4}}, \angle u_{1} p z_{1}=\arcsin \frac{1}{2 \rho} .
$$

Thus, in the right triangle $p q z_{1}$, we have

$$
\angle z_{1} p q=\angle u_{1} p q+\angle u_{1} p z_{1}=\frac{\pi}{6}+\arcsin \frac{1}{2 \rho},
$$

and hence

$$
\begin{aligned}
\|p q\| & =\left\|p z_{1}\right\| \cos \angle z_{1} p q \\
& =\sqrt{\rho^{2}-\frac{1}{4}} \cos \left(\frac{\pi}{6}+\arcsin \frac{1}{2 \rho}\right) \\
& =h(\rho) .
\end{aligned}
$$


In addition, $\left\|z_{1} q\right\|=g(\rho)$, where

$$
g(\rho)=\sqrt{\rho^{2}-\frac{1}{4}} \sin \left(\frac{\pi}{6}+\arcsin \frac{1}{2 \rho}\right) .
$$

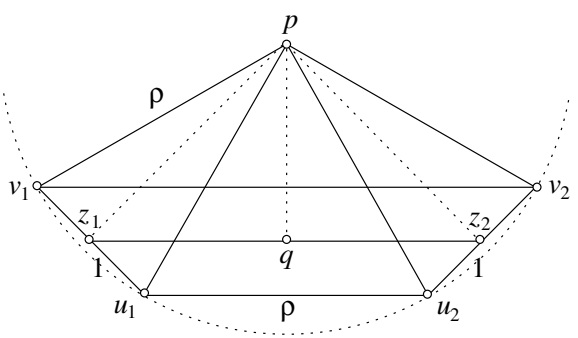

Fig. 1. The distance between $p$ and $z_{1} z_{2}$ is exactly $h(\rho)$.

From the above geometric interpretation, we immediately have $h(\rho)<\frac{\sqrt{3}}{2} \rho$. From the algebraic expression of $h(\rho)$, it is easy to see that $h(\rho)$ strictly increases with $\rho$ and hence

$$
h(\rho) \geq h(1)=\frac{\sqrt{3}}{4} .
$$

A straightforward calculation yields

$$
g(\rho)=\frac{1}{2} \sqrt{1-\frac{1}{4 \rho^{2}}}\left(\sqrt{\rho^{2}-\frac{1}{4}}+\frac{\sqrt{3}}{2}\right) .
$$

So, $g(\rho)$ also strictly increases with $\rho$, and hence

$$
g(\rho) \geq g(1)=\frac{3}{4} .
$$

Consider a horizontal strip $S$ of height $h(\rho)$, and let $L_{1}$ and $L_{2}$ be its top boundary and bottom boundary respectively. Suppose that $u$ and $v$ are two points. We introduce three (closed) rectangles with two sides aligned with the vertical lines through $u$ and $v$ respectively.

- $S(u, v)$ : the portion of $S$ between the two vertical lines through $u$ and $v$.

- $R(u, v)$ : the rectangle surrounded by the vertical lines through $u$ and $v$ respectively and the two horizontal lines through $u$ and $v$ respectively. Note that $u v$ is a diagonal of $R(u, v)$.

- $T(u, v)$ : the rectangle whose top side is the upper one of the top sides $R(u, v)$ and $S(u, v)$, and whose bottom sides are the lower one of the bottom sides of $R(u, v)$ and $S(u, v)$. Clearly, it is the minimal rectangle with vertical and horizontal sides which contains $S(u, v)$ and $R(u, v)$.

We begin with two technical geometric lemmas, whose proofs are omitted due the space limitation.

Lemma 3: Suppose that $u v$ is a line segment satisfying that its length is at most one and its midpoint $z$ is in $S$.
1) Let $p$ and $q$ be the intersection points of $l_{z}$ with $L_{1}$ and $L_{2}$ respectively. Let $\Omega$ be the intersection of the two $\sqrt{\rho^{2}-\frac{1}{4}}$-disks centered at $p$ and $q$ respectively (see Figure 2(a)). Then, $\Omega \subseteq B_{\rho}(u, v)$ and the length of the line segment $\Omega \cap L_{1}$ is $2 g(\rho)$.

2) Suppose that $u$ is below $L_{1}$. Let $p_{1}$ and $p_{2}$ be the two points on $L_{1}$ such that $\triangle u p_{1} p_{2}$ is regular (see Figure 2(b)). Then, the rectangle $R\left(u, p_{1}\right) \cup R\left(u, p_{2}\right) \subseteq$ $B_{\rho}(u, v)$.

3) $T(u, v) \subseteq B_{\rho}(u, v)$.

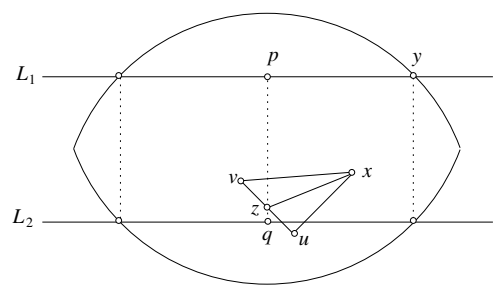

(a)

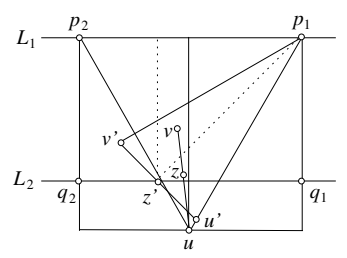

(b)
Fig. 2. Two subregions of $B_{\rho}(u, v)$.

Lemma 4: Consider two line segments $u_{i} v_{i}$ for $1 \leq i \leq 2$ of length at most one whose midpoints lying in $S$ from the left to right. If $\left\|u_{1} u_{2}\right\| \leq \rho$ and $u_{1}$ lies to the left of $u_{2}$, then

$$
T\left(u_{1}, u_{2}\right) \subseteq B_{\rho}\left(u_{1}, v_{1}, u_{2}, v_{2}\right) .
$$

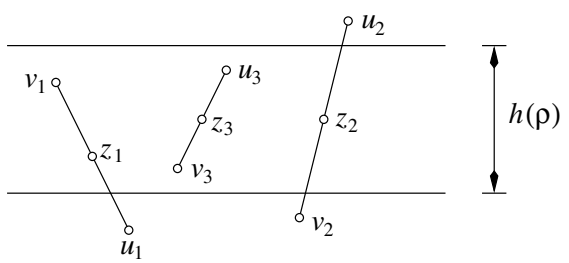

Fig. 3. If $u_{1} v_{1}$ and $u_{3} v_{3}$ conflict with each other, then at least one of them conflicts with $u_{2} v_{2}$.

Now, we are ready to prove Theorem 1 . For each $1 \leq i \leq 3$, let $u_{i}$ and $v_{i}$ be the two endpoints of $a_{i}$ and $z_{i}$ be the midpoint of $a_{i}$ (see Figure 3). We first claim that both $u_{3}$ and $v_{3}$ lie on the right side of $l_{z_{1}}$. Assume to the contrary that our claim does not hold. Since $z_{3}$ lies to the right of $z_{1}, u_{3}$ and $v_{3}$ lie on different sides of $z_{1}$. By symmetry, we assume that $u_{3}$ is on the left side of $z_{1}$, and $u_{3}$ is below $v_{3}$ (see Figure 4). Then, $u_{3}$ is below $L_{1}$. Since the distance between $u_{3}$ and $l_{z_{1}}$ is at most $1 / 2, u_{3}$ cannot be in $S$. Hence, $u_{3}$ is below $L_{2}$. Let $q$ be the intersection point of $l_{z_{1}}$ and $L_{2}$. Then,

$$
\begin{aligned}
\left\|q u_{3}\right\| & >\sqrt{\rho^{2}-\frac{1}{4}}-h(\rho) \\
& =\sqrt{\rho^{2}-\frac{1}{4}}\left(1-\cos \left(\frac{\pi}{6}+\arcsin \frac{1}{2 \rho}\right)\right) .
\end{aligned}
$$


Hence,

$$
\begin{aligned}
\left\|q z_{3}\right\|^{2} & \leq\left\|u_{3} z_{3}\right\|^{2}-\left\|q u_{3}\right\|^{2} \\
& <\frac{1}{4}-\left(\rho^{2}-\frac{1}{4}\right)\left(1-\cos \left(\frac{\pi}{6}+\arcsin \frac{1}{2 \rho}\right)\right)^{2} \\
& =\frac{1}{4}-\left(1-\frac{1}{4 \rho^{2}}\right)\left(\rho-\frac{\sqrt{3}}{2} \sqrt{\rho^{2}-\frac{1}{4}}+\frac{1}{4}\right)^{2} .
\end{aligned}
$$

The last expression decreases with $\rho$ when $\rho \geq 1$ because both $1-\frac{1}{4 \rho^{2}}$ and $\rho-\frac{\sqrt{3}}{2} \sqrt{\rho^{2}-\frac{1}{4}}+\frac{1}{4}$ are positive and increase with $\rho$. Hence, the last expression achieves its maximum $1 / 16$ at $\rho=1$. So, $\left\|q z_{3}\right\|<1 / 4$. On the other hand, since $v_{3}$ must be above $L_{2},\left\|q v_{3}\right\|>g(\rho)$. Therefore,

$$
\left\|v_{3} z_{3}\right\| \geq\left\|q v_{3}\right\|-\left\|q z_{3}\right\|>g(\rho)-\frac{1}{4} \geq \frac{3}{4}-\frac{1}{4}=\frac{1}{2},
$$

which is a contradiction. So, our claim holds. Similarly, we can show that both $u_{3}$ and $v_{3}$ lie on left side of $l_{z_{2}}$. So, both $u_{3}$ and $v_{3}$ lie between $l_{z_{1}}$ and $l_{z_{2}}$.

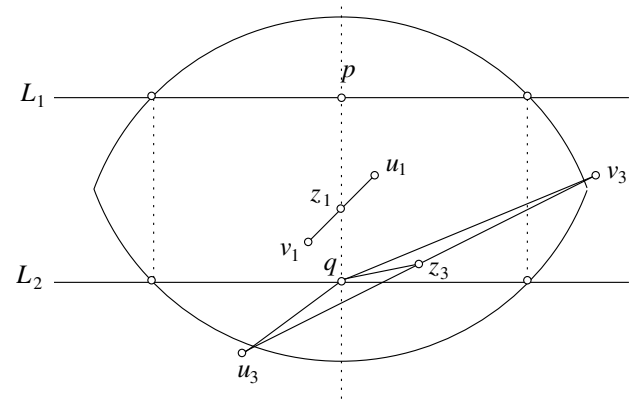

Fig. 4. If $u_{3}$ and $v_{3}$ lie on different sides of $l_{z_{1}}$, then $\left\|u_{3} v_{3}\right\|>1$.

Next, we claim that both $u_{1}$ and $v_{1}$ are apart from $l_{z_{2}}$ by a distance greater than $1 / 2$. Assume to the contrary that our claim does not hold. By symmetry, we assume that the distance between $u_{1}$ and $l_{z_{2}}$ is at most $1 / 2$. Then, the distance between $l_{z_{1}}$ and $l_{z_{2}}$ is at most one. By Lemma 3(1),

$$
S\left(z_{1}, z_{2}\right) \subseteq B_{\rho}\left(u_{1}, v_{1}\right) \cup B_{\rho}\left(u_{2}, v_{2}\right) \subseteq B_{\rho}\left(u_{1}, v_{1}, u_{2}, v_{2}\right) .
$$

Thus, $u_{3}$ and $v_{3}$ lie in the two pieces of the vertical strip between $l_{z_{1}}$ and $l_{z_{2}}$ separated by $B_{\rho}\left(u_{1}, v_{1}, u_{2}, v_{2}\right)$. On the other hand, both chords $B_{\rho}\left(u_{1}\right) \cap l_{z_{1}}$ and $B_{\rho}\left(u_{1}\right) \cap l_{z_{2}}$ have length at least $\sqrt{3}$. Hence, $\left\|u_{3} v_{3}\right\| \geq \sqrt{3}$, which is a contradiction. Thus, our claim holds. Similarly, we can show that both $u_{2}$ and $v_{2}$ are apart from $l_{z_{1}}$ by a distance greater than $1 / 2$. So, both $u_{1}$ and $v_{1}$ lie to the left side of both $u_{2}$ and $v_{2}$.

Finally, we prove by contradiction that $a_{1}$ and $a_{2}$ are independent. Assume to the contrary that $a_{1}$ and $a_{2}$ are not independent. By symmetry, we assume that $\left\|u_{1} u_{2}\right\| \leq \rho$. By Lemma 3 and Lemma 4,

$$
T\left(u_{1}, v_{1}\right) \cup T\left(u_{1}, u_{2}\right) \cup T\left(u_{2}, v_{2}\right)
$$

contains $S\left(z_{1}, z_{2}\right)$ and is singly-connected (i.e., contains no holes). So, $u_{3}$ and $v_{3}$ lie in the two pieces of the vertical strip between $l_{z_{1}}$ and $l_{z_{2}}$ separated by

$$
T\left(u_{1}, v_{1}\right) \cup T\left(u_{1}, u_{2}\right) \cup T\left(u_{2}, v_{2}\right) .
$$

Hence, $u_{3} v_{3}$ must cross the path $v_{1} u_{1} u_{2} v_{2}$ consisting of the three line segments $v_{1} u_{1}, u_{1} u_{2}$ and $u_{2} v_{2}$. We consider three cases:

Case 1: $u_{3} v_{3}$ crosses $v_{1} u_{1}$. Then,

$$
\left\|u_{1} u_{3}\right\|+\left\|v_{1} v_{3}\right\| \leq\left\|u_{1} v_{1}\right\|+\left\|u_{3} v_{3}\right\| \leq 2 .
$$

Hence,

$$
\min \left\{\left\|u_{1} u_{3}\right\|,\left\|v_{1} v_{3}\right\|\right\} \leq 1 \leq \rho,
$$

which is a contradiction.

Case 2: $u_{3} v_{3}$ crosses $u_{2} v_{2}$. Using the same argument as in Case 1, we can reach a contradiction.

Case 3: $u_{3} v_{3}$ crosses $u_{1} u_{2}$. Then,

$$
\left\|u_{1} u_{3}\right\|+\left\|u_{2} v_{3}\right\| \leq\left\|u_{1} u_{2}\right\|+\left\|u_{3} v_{3}\right\| \leq \rho+1 .
$$

Hence,

$$
\min \left\{\left\|u_{1} u_{3}\right\|,\left\|u_{2} v_{3}\right\|\right\} \leq \frac{\rho+1}{2} \leq \rho,
$$

which is a contradiction.

Therefore, in either case, we get a contradiction. This completes the proof of Theorem 1 .

\section{B. Proof of Theorem 2}

The function $h(\rho)$ defined in Theorem 2 has the following geometric interpretation. Consider a convex quadruple puqv in which $\angle q u v=\frac{\pi}{2},\|q u\|=1,\|q v\|=\|q p\|=\rho$ and $\|p v\|=\rho-1$ (see Figure 5). Then, the distance between $p$ and $u v$ is exactly $h(\rho)$. Such geometric interpretation immediately implies that

$$
2 \sqrt{(\rho-1)^{2}-h(\rho)^{2}} \leq \sqrt{\rho^{2}-1}
$$

and the equality holds if and only if $\rho=1$. Indeed, when $\rho=1, p$ is identical to $v$ and hence $h(\rho)=0$, which implies both sides of the above inequality equal to zero. When $\rho>1$, we have

$$
\|p u\|>\|p q\|-\|q u\|=\rho-1=\|p v\|,
$$


which implies

$$
\begin{aligned}
& \sqrt{\rho^{2}-1}=\|u v\| \\
& =\sqrt{\|p u\|^{2}-h(\rho)^{2}}+\sqrt{\|p v\|^{2}-h(\rho)^{2}} \\
& >2 \sqrt{(\rho-1)^{2}-h(\rho)^{2}} .
\end{aligned}
$$

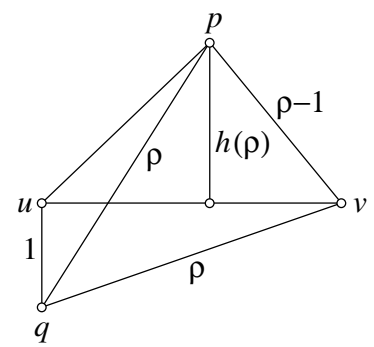

Fig. 5. The distance between $p$ and $u v$ is exactly $h(\rho)$

Clearly, $h(\rho)$ is continuous. It was proved in [8] that $\arccos \frac{\rho-1}{2 \rho}-\arcsin \frac{1}{\rho}$ strictly increases with $\rho$. Thus, $h(\rho)$ is also strictly increasing. Since

$$
\lim _{\rho \rightarrow \infty}\left(\arccos \frac{\rho-1}{2 \rho}-\arcsin \frac{1}{\rho}\right)=\frac{\pi}{3} .
$$

we have

$$
\arccos \frac{\rho-1}{2 \rho}-\arcsin \frac{1}{\rho}<\frac{\pi}{3} .
$$

Thus, when $\rho>1$,

$$
\begin{aligned}
h(\rho) & =(\rho-1) \sin \left(\arccos \frac{\rho-1}{2 \rho}-\arcsin \frac{1}{\rho}\right) \\
& <(\rho-1) \sin \frac{\pi}{3}=\frac{\sqrt{3}}{2}(\rho-1) .
\end{aligned}
$$

So,

$$
h(\rho) \leq \frac{\sqrt{3}}{2}(\rho-1)
$$

and the equality holds if and only if $\rho=1$.

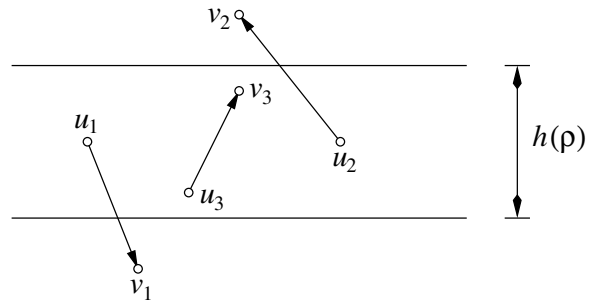

Fig. 6. If both $\left(u_{1}, v_{1}\right)$ and $\left(u_{2}, v_{2}\right)$ are independent with $\left(u_{3}, v_{3}\right)$, then they are independent with each other.

Now, we are ready to prove Theorem 2 . For each $1 \leq i \leq 3$, let $a_{i}=\left(u_{i}, v_{i}\right)$. It is easy to prove that Theorem 2 holds when $\rho=1$. So, we assume that $\rho>1$. Since both $a_{1}$ and $a_{2}$ are independent with $a_{3},\left\|u_{1} u_{3}\right\|>\rho-1$ and $\left\|u_{2} u_{3}\right\|>\rho-1$.
Since $h(\rho)<\frac{\sqrt{3}}{2}(\rho-1)$, the three vertical lines $l_{u_{i}}$ for $1 \leq$ $i \leq 3$ are distinct. Let $w$ be the intersection point between the segment $u_{1} u_{2}$ and $l_{u_{3}}$. Then,

$$
\left\|u_{3} w\right\| \leq h(\rho)<\frac{\sqrt{3}}{2}(\rho-1) .
$$

So $u_{3} w$ is shorter than both $u_{1} u_{3}$ and $u_{2} u_{3}$, which implies that both $\angle u_{3} u_{1} u_{2}$ and $\angle u_{3} u_{2} u_{1}$ are acute. Furthermore, the distance between $u_{3}$ and $u_{1} u_{2}$ is no more than $\left\|u_{3} w\right\|$, and hence is at most $h(\rho)$. Thus,

$$
\left\|u_{1} u_{2}\right\|>2 \sqrt{(\rho-1)^{2}-h(\rho)^{2}}>\rho-1 .
$$

For $i=1$ and 2 , let $p_{i}$ be the point on $\partial B_{\rho-1}\left(u_{i}\right)$ satisfying that (1) the distance between $p_{i}$ and the line $u_{1} u_{2}$ is $h(\rho)$, (2) $p_{i}$ lies on the same side of $u_{1} u_{2}$ as $u_{3}$, and (3) the perpendicular foot $p_{i}^{\prime}$ of $p_{i}$ on the line $u_{1} u_{2}$ is on the segment $u_{1} u_{2}$ (see Figure 7). Since

$$
\left\|u_{1} u_{2}\right\|>2 \sqrt{(\rho-1)^{2}-h(\rho)^{2}}=\left\|u_{1} p_{1}^{\prime}\right\|+\left\|u_{2} p_{2}^{\prime}\right\|,
$$

$p_{1}^{\prime}$ is closer to $u_{1}$ than $p_{2}^{\prime}$. Thus,

$$
\left\|u_{1} p_{2}\right\|>\left\|u_{1} p_{1}\right\|=\rho-1 .
$$

This implies that $p_{2}$ is outside $B_{\rho-1}\left(u_{1}\right)$. Similarly, $p_{1}$ is outside $B_{\rho-1}\left(u_{2}\right)$. Then, $u_{3}$ lies inside the rectangle $p_{1} p_{1}^{\prime} p_{2}^{\prime} p_{2}$.

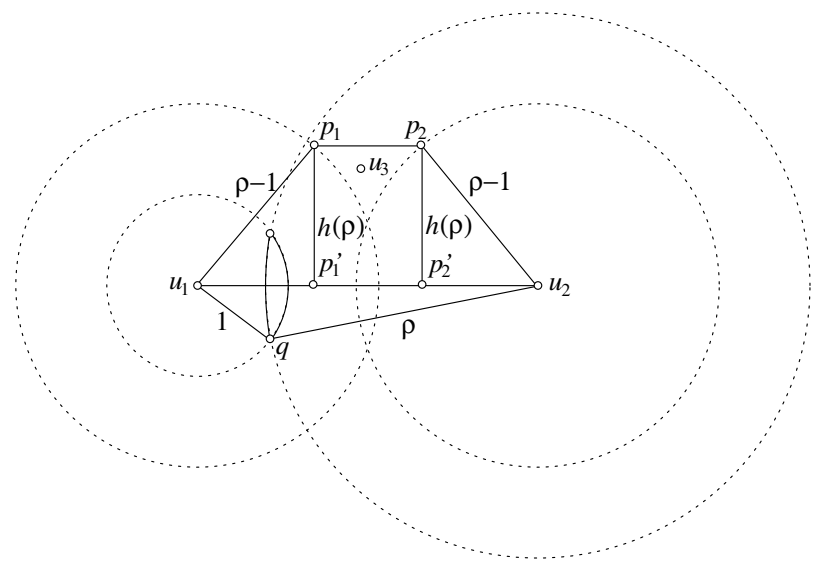

Fig. 7. Figure for the proof of Theorem 2.

Next, we claim that

$$
B_{1}\left(u_{1}\right) \cap B_{\rho}\left(u_{2}\right) \subset B_{\rho}\left(u_{3}\right) .
$$

The claim holds trivially if $\left\|u_{1} u_{2}\right\|>\rho+1$. So, we assume that $\left\|u_{1} u_{2}\right\| \leq \rho+1$. Since $\left\|u_{1} u_{2}\right\|>\rho-1, \partial B_{1}\left(u_{1}\right)$ and $\partial B_{\rho}\left(u_{2}\right)$ intersect at two (possibly identical) points. Let $q$ be the intersection point of $\partial B_{1}\left(u_{1}\right)$ and $\partial B_{\rho}\left(u_{2}\right)$ which lies on different side of $u_{1} u_{2}$ from $u_{3}$ (see Figure 7). Consider the triangle $\triangle p_{2} q u_{2}$. Since $p_{2} u_{2}$ and $q u_{2}$ have fixed length, $\left\|p_{2} q\right\|$ strictly increases with $\angle p_{2} u_{2} q$ by the law of cosine. 
Since $\angle p_{2} u_{2} q=\angle p_{2} u_{2} u_{1}+\angle q u_{2} u_{1}$ and $\angle p_{2} u_{2} u_{1}$ is also fixed, $\left\|p_{2} q\right\|$ also strictly increases with $\angle q u_{2} u_{1}$. Since

$$
2 \sqrt{(\rho-1)^{2}-h(\rho)^{2}} \leq \sqrt{\rho^{2}-1}
$$

Thus, $\angle q u_{2} u_{1}$, and hence $\left\|p_{2} q\right\|$, achieves its maximum when $q u_{1}$ is perpendicular to $u_{1} u_{2}$. By the geometric interpretation of $h(\rho),\left\|p_{2} q\right\|=\rho$ when $q u_{1}$ is perpendicular to $u_{1} u_{2}$. Thus, $\left\|p_{2} q\right\| \leq \rho$ in general. Since both $q$ and $p_{1}$ lie on the same side of the perpendicular bisector of $u_{1} u_{2}$, which is also the perpendicular bisector of $p_{1} p_{2}$, we have

$$
\left\|p_{1} q\right\| \leq\left\|p_{2} q\right\| \leq \rho
$$

Clearly, for $i=1$ and 2 ,

$$
\left\|p_{i}^{\prime} q\right\| \leq \max \left\{\left\|q u_{1}\right\|,\left\|q u_{2}\right\|\right\}=\rho .
$$

Thus, the distances from $q$ to the four vertices of the rectangle $p_{1} p_{1}^{\prime} p_{2}^{\prime} p_{2}$ are all at most $\rho$. So, $\left\|q u_{3}\right\| \leq \rho$ and hence

$$
B_{1}\left(u_{1}\right) \cap B_{\rho}\left(u_{2}\right) \subset B_{\rho}\left(u_{3}\right) .
$$

So, our claim holds. Similarly, we can show that

$$
B_{1}\left(u_{2}\right) \cap B_{\rho}\left(u_{1}\right) \subset B_{\rho}\left(u_{3}\right) .
$$

Finally, we prove by contradiction that $a_{1}$ and $a_{2}$ are independent. Assume to the contrary that $a_{1}$ and $a_{2}$ are not independent. Then, either $v_{1} \in B_{1}\left(u_{1}\right) \cap B_{\rho}\left(u_{2}\right)$, or $v_{2} \in B_{1}\left(u_{2}\right) \cap B_{\rho}\left(u_{1}\right)$. By symmetry, we assume the former holds. Then, $v_{1} \in B_{\rho}\left(u_{3}\right)$. Hence, $a_{3}$ conflicts with $a_{1}$, which is a contradiction. This completes the proof of Theorem 2.

\section{Approximate CApAcity Subregions}

In this section, we exploit the strip-wise transitivity of independence under either the 802.11 interference model or the protocol interference model to construct approximate capacity subregions of uniform multihop wireless networks under either the 802.11 interference model or the protocol interference model. We assume that all nodes have unit communication radius, and have interference radius equal to $\rho$. Under the 802.11 interference model, the height function $h(\rho)$ is as defined in Theorem 1; under the protocol interference model, we assume that $\rho>1$ and the height function $h(\rho)$ is as defined in Theorem 2 . We define the representative of the communication links as follows. Under the 802.11 interference model, the representative of a link is its midpoint; under the protocol interference model, the representative of a link is its transmitting endpoint. A link is said to be associated with a horizontal strip if its representative lies in this strip.

Now, we describe the construction of a polynomial $\mu$ approximate capacity subregion $Q$, where

$$
\mu=\left\lceil\frac{\rho+1}{h(\rho)}\right\rceil+1 .
$$

We first compute the minimal axis-parallel rectangle surrounding all the networking nodes. Then, we partition such rectangle into top-closed bottom-open horizontal strips in the manner that the upper boundary of the top-most strip aligns with the top of the rectangle, the heights of all strips except the bottom-most one are all equal to $(\rho+1) /(\mu-1)$, and the height of the bottom-most strip is at most $(\rho+1) /(\mu-1)$ (see Figure 8 ). Let $\ell$ denote the total number of strips. We number the successive strips from top to bottom using integers $0,1, \cdots, \ell-1$, and let $A_{i}$ denote the set of links in $A$ associated with the strip $i$. Then, $A_{0}, A_{1}, \cdots, A_{\ell-1}$ form a partition of $A$. For each $0 \leq i \leq \ell-1$, let $G_{i}$ be the conflict graph of $A_{i}$. By Theorem 1 and Theorem 2, each $G_{i}$ is a cocomparability graph with the lexicographic ordering of the representatives of $A_{i}$ being its cocomparability ordering. Let $P_{i}$ be the independence polytope of $G_{i}$ as constructed in Section II. We define $Q$ to be $\frac{1}{\mu} \prod_{i=0}^{\ell-1} P_{i}$.

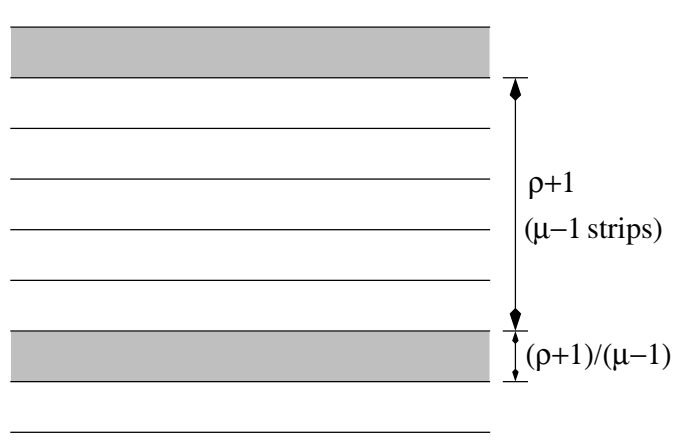

Fig. 8. Partition of the plane into half-open half-closed strips of height $(\rho+1) /(\mu-1)$ where $\mu=\lceil(\rho+1) / h(\rho)\rceil+1$. Two strips separated by $\mu-1$ strips can spatially share the time-slots.

Next, we show that $Q$ is indeed a polynomial $\mu$-approximate capacity subregion. Let $P$ be the capacity region. It is trivial that

$$
P \subseteq \prod_{i=0}^{\ell-1} P_{i}=\mu Q
$$

On the other hand, we give a polynomial algorithm which produces a fractional schedule of length at most one for any $d \in Q$. As a result, $Q \subseteq P$ and hence $Q$ is a polynomial $\mu$-approximation of $P$. For each $0 \leq i<\ell$, let $G_{i}^{*}$ be the augmented complementary digraph of $G_{i}$, and $\mathcal{F}_{i}^{*}$ denote the set of flows from the auxiliary source to the the auxiliary sink in $G_{i}^{*}$. Any $d \in Q$ is represented by $\ell$ flows $f_{i} \in \mathcal{F}_{i}^{*}$ with $\operatorname{val}\left(f_{i}\right) \leq 1 / \mu$ for $0 \leq i<\ell$ such that the restriction of $d$ on $A_{i}$ is $d_{f_{i}}$ for $0 \leq i<\ell$. Using the method described in Section II, we can obtain in polynomial time a link schedule $\Pi_{i}$ for $d_{f_{i}}$ of length $\operatorname{val}\left(f_{i}\right)$, which is at most $1 / \mu$. Note that any pair of links $a \in A_{i}$ and $a^{\prime} \in A_{i^{\prime}}$ are independent if $0 \leq i<i^{\prime}<\ell$ and $i=i^{\prime}(\bmod \mu)$. Thus, for each $0 \leq j<\mu$ we can merge all link schedules $\Pi_{i}$ with $0 \leq i<\ell$ and $i=j \bmod \mu$ into 
a link schedule of length at most $1 / \mu$. The concatenation of these $\mu$ link schedules is a link schedule for $d$ of length at most $\mu \cdot(1 / \mu)=1$.

In the remaining of this section, we compute the value of $\mu$. We first compute $\mu$ under the 802.11 interference model. Note that

$$
\frac{\rho+1}{h(\rho)}=\frac{1+\frac{1}{\rho}}{\sqrt{1-\frac{1}{4 \rho^{2}}} \cos \left(\frac{\pi}{6}+\arcsin \frac{1}{2 \rho}\right)} .
$$

Since $1+\frac{1}{\rho}$ strictly decreases with $\rho$ and both $\sqrt{1-\frac{1}{4 \rho^{2}}}$ and $\cos \left(\frac{\pi}{6}+\arcsin \frac{1}{2 \rho}\right)$ strictly increase with $\rho, \frac{\rho+1}{h(\rho)}$ strictly decreases with $\rho$. When $\rho=1$,

$$
\frac{\rho+1}{h(\rho)}=\frac{2}{\frac{\sqrt{3}}{4}}=\frac{8}{\sqrt{3}} \text {. }
$$

On the other hand,

$$
\lim _{\rho \rightarrow \infty} \frac{\rho+1}{h(\rho)}=\frac{1}{\cos \frac{\pi}{6}}=\frac{2}{\sqrt{3}} .
$$

Hence,

$$
\frac{2}{\sqrt{3}}<\frac{\rho+1}{h(\rho)} \leq \frac{8}{\sqrt{3}},
$$

which implies that $\mu-1$ is an integer between 2 and 5 . The root of $\frac{\rho+1}{h(\rho)}=4$ (respectively, 3 and 2 ) in $[1, \infty)$ can be translated as a root of a quartic equation and is equal to 1.0891 (respectively, 1.3609 and 2.2907 ) by applying the quartic formula. Therefore,

$$
\mu= \begin{cases}6 & \text { if } \rho \in[1,1.0891) ; \\ 5 & \text { if } \rho \in[1.0891,1.3609) \\ 4 & \text { if } \rho \in[1.3609,2.2907) \\ 3 & \text { if } \rho \in[2.2907, \infty) .\end{cases}
$$

Next, we compute $\mu$ under the protocol interference model. Note that

$$
\frac{\rho+1}{h(\rho)}=\frac{\rho+1}{\rho-1} \cdot \frac{1}{\sin \left(\arccos \frac{\rho-1}{2 \rho}-\arcsin \frac{1}{\rho}\right)} .
$$

Since $\frac{\rho+1}{\rho-1}=1+\frac{2}{\rho-1}$ strictly decreases with $\rho$ and $\arccos \frac{\rho-1}{2 \rho}-\arcsin \frac{1}{\rho}$ strictly increases with $\rho[8], \frac{\rho+1}{h(\rho)}$ strictly decreases with $\rho$. Since $h(\rho)<\frac{\sqrt{3}}{2}(\rho-1)$, we have

$$
\frac{\rho+1}{h(\rho)}>\frac{\rho+1}{\frac{\sqrt{3}}{2}(\rho-1)}>\frac{2}{\sqrt{3}} \text {. }
$$

Let $\rho_{1}=\infty$, and for each integer $k \geq 2$ let $\rho_{k}$ be the unique root of $(\rho+1) / h(\rho)=k$ in $[1, \infty)$. Then, $\mu=k+1$ over $\left[\rho_{k}, \rho_{k-1}\right)$ for any $k \geq 2$. By a straightforward algebraic calculation, $\rho_{k}$ is the unique root of the following quartic polynomial in $(1, \infty)$ :

$$
\begin{aligned}
& \left(4-3 k^{2}\right) \rho^{4}+4\left(k^{2}+k+2\right) \rho^{3}+2\left(3 k^{2}-2 k+2\right) \rho^{2} \\
& -4 k(3 k+1) \rho+\left(5 k^{2}+4 k\right) .
\end{aligned}
$$

The numeric values of $\rho_{k}$ can be computed with the quartic formula. Table I lists the numeric values of $\rho_{k}$ for $2 \leq k \leq 11$.

\begin{tabular}{|c|c||c|c|}
\hline$k$ & $\rho_{k}$ & $k$ & $\rho_{k}$ \\
\hline \hline 2 & 4.2462 & 7 & 1.5715 \\
\hline 3 & 2.5689 & 8 & 1.5009 \\
\hline 4 & 2.0632 & 9 & 1.4476 \\
\hline 5 & 1.8167 & 10 & 1.4058 \\
\hline 6 & 1.6697 & 11 & 1.3721 \\
\hline
\end{tabular}

TABLE I

NUMBERIC VALUES OF $\rho_{k}$ FOR $2 \leq k \leq 11$.

\section{Maximum (CONCURREnt) Multiflow}

Consider a wireless network $\mathbf{N}$ given by a triple $(V, A, \mathcal{I})$ and let $P$ denote its capacity region. Suppose that we are given $k$ commodities with $s_{j}, t_{j}$ being the source and sink, respectively, for commodity $j$. We use $\mathcal{F}_{j}$ to denote the set of $s_{j}-t_{j}$ flows in the digraph $(V, A)$. A $k$-flow is a sequence of flows $\left\langle f_{1}, f_{2}, \cdots, f_{k}\right\rangle$ with $f_{j} \in \mathcal{F}_{j}$ for each $1 \leq i \leq k$. The maximum multiflow is defined by the following linear program (LP):

$$
\begin{array}{ll}
\max & \sum_{j=1}^{k} \operatorname{val}\left(f_{j}\right) \\
\text { s.t. } & f_{j} \in \mathcal{F}_{j}, \forall 1 \leq j \leq k ; \\
& \sum_{j=1}^{k} f_{j} \in P .
\end{array}
$$

Similarly, the maximum concurrent multiflow with demands $d(j)$ for $1 \leq j \leq k$ is defined by the following LP:

$$
\begin{array}{ll}
\max & \phi \\
\text { s.t. } & f_{j} \in \mathcal{F}_{j}, \forall 1 \leq j \leq k ; \\
& \operatorname{val}\left(f_{j}\right) \geq \phi d(j), \forall 1 \leq j \leq k ; \\
& \sum_{j=1}^{k} f_{j} \in P .
\end{array}
$$

However, the membership of the capacity region $P$ is NPcomplete in general [7].

For developing a practical $\mu$-approximation algorithm, we replace the capacity region $P$ by a polynomial $\mu$-approximate capacity subregion $Q$. Suppose that $Q$ is a polynomial $\mu$ approximate capacity subregion. A $k$-flow $\left\langle f_{1}, f_{2}, \cdots, f_{k}\right\rangle$ is said to be $Q$-restricted if $\sum_{j=1}^{k} f_{j} \in Q$. The maximum $Q$ restricted multiflow is defined by the following LP:

$$
\begin{array}{ll}
\max & \sum_{j=1}^{k} \operatorname{val}\left(f_{j}\right) \\
\text { s.t. } & f_{j} \in \mathcal{F}_{j}, \forall 1 \leq j \leq k ; \\
& \sum_{j=1}^{k} f_{j} \in Q .
\end{array}
$$

This LP is of polynomial size and we solve this LP in polynomial time to obtain a $k$-flow $\left\langle f_{1}, f_{2}, \cdots, f_{k}\right\rangle$. Then we compute a fractional link schedule of length at most one for $\sum_{j=1}^{k} f_{j}$. Such link schedule is a $\mu$-approximate solution. Similarly, the maximum concurrent $Q$-restricted multiflow 
with demands $d(j)$ for $1 \leq j \leq k$ is defined by the following LP:

$$
\begin{array}{ll}
\max & \phi \\
\text { s.t. } & f_{j} \in \mathcal{F}_{j}, \forall 1 \leq j \leq k \\
& \operatorname{val}\left(f_{j}\right) \geq \phi d(j), \forall 1 \leq j \leq k ; \\
& \sum_{j=1}^{k} f_{j} \in Q .
\end{array}
$$

We first solve this LP of polynomial size in polynomial time to obtain a $k$-flow $\left\langle f_{1}, f_{2}, \cdots, f_{k}\right\rangle$, and then compute a fractional link schedule of length at most one for $\sum_{j=1}^{k} f_{j}$. This link schedule is a $\mu$-approximate solution.

Let $Q$ be the approximate capacity subregion defined in the previous section. We describe an actual implementation of computing the maximum (concurrent) $Q$-restricted multiflow. We compute $\mu$ and a partition $A_{0}, A_{1}, \cdots, A_{\ell-1}$ of $A$ as in the previous section. For each $0 \leq i<\ell$, let $G_{i}$ be the conflict graph of $A_{i}$. Then, each $G_{i}$ is a cocomparability graph with the lexicographic ordering of the representatives of $A_{i}$ being its cocomparability ordering. Let $G_{i}^{*}$ be the augmented complementary digraph of $G_{i}$, and $\mathcal{F}_{i}^{*}$ denote the set of flows from the auxiliary source to the auxiliary sink in $G_{i}^{*}$. Then, the maximum $Q$-restricted multiflow can be rewritten as the following LP:

$$
\begin{array}{ll}
\max & \sum_{j=1}^{k} \operatorname{val}\left(f_{j}\right) \\
\text { s.t. } & f_{j} \in \mathcal{F}_{j}, \forall 1 \leq j \leq k ; \\
& f_{i}^{*} \in \mathcal{F}_{i}^{*}, \forall 0 \leq i<\ell ; \\
& \operatorname{val}\left(f_{i}^{*}\right) \leq 1 / \mu, \forall 0 \leq i<\ell ; \\
& \sum_{j=1}^{k} f_{j}(e)=f_{i}^{*}\left(\delta_{G_{i}^{*}}^{\text {out }}(a)\right), \forall a \in A_{i}, \forall 0 \leq i<\ell .
\end{array}
$$

We remark that two groups of flows $\left\{f_{1}, f_{2}, \cdots, f_{k}\right\}$ and $\left\{f_{0}^{*}, f_{1}^{*}, \cdots, f_{\ell-1}^{*}\right\}$ are involved in this LP. We solve this LP and obtain these two groups of flows. A link schedule of length at most one for $\sum_{j=1}^{k} f_{j}$ is then constructed directly from the second group of flows $\left\{f_{0}^{*}, f_{1}^{*}, \cdots, f_{\ell-1}^{*}\right\}$ using the algorithm described in Section IV. Similarly, the maximum concurrent $Q$-restricted multiflow with demands $d(j)$ for $1 \leq j \leq k$ can be rewritten as the following LP:

$$
\begin{array}{ll}
\max & \phi \\
\text { s.t. } & f_{j} \in \mathcal{F}_{j}, \forall 1 \leq j \leq k ; \\
& f_{i}^{*} \in \mathcal{F}_{i}^{*}, \forall 0 \leq i<\ell ; \\
& \operatorname{val}\left(f_{j}\right) \geq \phi d(j), \forall 1 \leq j \leq k ; \\
& \operatorname{val}\left(f_{i}^{*}\right) \leq 1 / \mu, \forall 0 \leq i<\ell ; \\
& \sum_{j=1}^{k} f_{j}(e)=f_{i}^{*}\left(\delta_{G_{i}^{*}}^{\text {out }}(a)\right), \forall a \in A_{i}, \forall 0 \leq i<\ell .
\end{array}
$$

By solving this LP, we obtain two groups of flows $\left\{f_{1}, f_{2}, \cdots, f_{k}\right\}$ and $\left\{f_{0}^{*}, f_{1}^{*}, \cdots, f_{\ell-1}^{*}\right\}$ and compute a link schedule for $\sum_{j=1}^{k} f_{j}$ from the second group of flows.

\section{CONCLUSION}

In this paper, we have discovered a nature of the wireless interference called strip-wise transitivity of independence under either the 802.11 interference model or the protocol interference model. We exploit such nature and utilize the independence polytopes of cocomparability graphs in a spatial-divide-conquer manner to construct a polynomial $\mu$ approximate capacity subregion, where $\mu$ decreases with $\rho$ in general and is smaller than the best-known ones obtained in [7]. For example, $\mu=3$ when $\rho \geq 2.2907$ under the 802.11 interference model or when $\rho \geq 4.2462$ under the protocol interference model. We also apply these polynomial $\mu$-approximate capacity subregions to compute $\mu$-approximate solutions for MMF and MCMF respectively.

\section{ACKNOWLEDGMENT}

Peng-Jun Wan and Lixin Wang were supported in part by the National Science Foundation of USA under grants CNS0831831 and CNS-0916666. Ai Huang and Minming Li were supported in part by a grant from the Research Grants Council of the Hong Kong Special Administrative Region, China, under Project No. CityU 117408. Frances Yao was partially supported by a grant from the Research Grants Council of the Hong Kong SAR, China, under Project No. CityU 122807, and the National Basic Research Program of China Grant 2007CB807900, 2007CB807901.

\section{REFERENCES}

[1] M. Alicherry, R. Bhatia, and L.E. Li, Joint Channel Assignment and Routing for Throughput Optimization in Multiradio Wireless Mesh Networks, IEEE Journal on Selected Areas in Communications 24(11):1960-1971 (2006). Also appeared in Proc. of ACM MobiCom 2005.

[2] P. Bácia, J.O. Cerdeira, Node packings on cocomparability graphs, Operations Research Letters 31(3): 341-342 (2003).

[3] C. Buragohain, S. Suri, C. D. Toth, and Y. Zhou, Improved throughput bounds for interference-aware routing in wireless networks, Proc. COCOON 2007, Lecture Notes in Computer Science 4598, 2007, pp. 210-221.

[4] L.R. Ford, and D.R. Fulkerson, Flows in Networks. Princeton University Press, Princeton 1962.

[5] M.C. Golumbic. Algorithmic graph theory and perfect graphs. Academic Press, New York, NY, 1980

[6] R.H. Möhring. Algorithmic aspects of comparability graphs and interval graphs. In I. Rival, editor, Graphs and Orders, pp. 41-101. Reidel, Dordrecht, 1985.

[7] P.-J. Wan, Multiflows in Multihop Wireless Networks, ACM MOBIHOC 2009, pp. 85-94.

[8] P.-J. Wan, X. Xu, L. Wang, X. Jia, and E.K. Park, Minimum-Latency Beaconing Schedule in Multihop Wireless Networks, IEEE INFOCOM 2009.

[9] Y. Wang, W. Wang, X.-Y. Li, and W.-Z. Song, Interference-Aware Joint Routing and TDMA Link Scheduling for Static Wireless Networks, IEEE Transactions on Parallel and Distributed Systems 19(12): 17091726 (2008). An early version of this paper appeared in Proc. of ACM MobiCom 2006. 